\title{
O modelo EEA para a investigação da emergência e desenvolvimento da comunicação e do self: bases conceituais e fundamentos teórico-metodológicos
}

\author{
Maria C. D. P. Lyra \\ Universidade Federal de Pernambuco
}

\begin{abstract}
Resumo
A coerência entre os fundamentos teóricos e metodológicos é requisito de toda investigação científica. Com o objetivo de ilustrar esta coerência, apresentamos, primeiramente, o modelo EEA, aplicado ao estudo do processo de desenvolvimento da comunicação mãe-bebê e à emergência do bebê como self dialógico. Este modelo integra uma seqüência de três padrões de organização denominados estabelecimento, extensão e abreviação (EEA). Em seguida, descrevemos, discutimos e justificamos as bases conceituais e as duas perspectivas teórico-metodológicas que fundamentam este modelo, Sistemas Dinâmicos e Dialogismo, sobretudo na perspectiva de Bakhtin.
\end{abstract}

Palavras-chave: processo de desenvolvimento; mãe-bebê; sistemas dinâmicos; dialogismo; self

\begin{abstract}
The EEA model for the investigation of communication and self emergence and development: conceptual and theoretical-methodological basis. Theoretical and methodological dimensions of scientific research need to be coherent. To illustrate such requirement we first present the EEA model, applied to the study of the process of development of mother-infant communication system and to the emergence of the dialogical self is studied. This model integrates three developmental patterns of organization of the mother-infant communication system: establishment, extension and abbreviation (EEA). Subsequently, we present, discuss and justify the conceptual basis and the two theoretical-methodological perspectives that give support for this model, Dynamic Systems and Dialogism according to Bakhtin.
\end{abstract}

Keywords: developmental process; mother-infant; dynamic systems; dialogism; self

$\mathrm{D}$ estacamos, neste artigo, a importância das questões de fundamento na área da Psicologia, em particular na Psicologia do Desenvolvimento. Mais ainda, propomos que a coerência entre os fundamentos teóricos e a metodologia adotada é requisito de toda investigação científica. Trata-se do que Valsiner (2006) chama de consistência vertical necessária à construção de todo conhecimento científico. Com o objetivo de ilustrar tal requisito, apresentamos, primeiramente, o modelo EEA, aplicado ao estudo do desenvolvimento do processo de comunicação mãe-bebê e à emergência do self dialógico. Em seguida, discutimos e justificamos as bases conceituais e as perspectivas dos Sistemas Dinâmicos e do Dialogismo (para o uso do termo dialogismo, ver Holquist, 1990), segundo Bakhtin (1986, 1993). Essas duas concepções nos fornecem os alicerces sobre os quais é construído o modelo EEA.

\section{$O$ modelo EEA}

O modelo EEA, estudando o processo de desenvolvimento da comunicação mãe-bebê, aborda as transformações que ocorrem no sistema de relações composto pela trocas mãe-bebê, ao longo da sua história de construção. Essas transformações são analisadas como construindo padrões sucessivos de organização desse sistema. O desenvolvimento da comunicação, concebido como diálogo, permite identificar a emergência e diferenciação do bebê como self dialógico.

Este modelo propõe uma seqüência de três padrões de organização - ou períodos de quase-estabilidade - do sistema de comunicação mãe-bebê, estabelecimento, extensão e abreviação (Lyra, 2000; no prelo a, b, c; Lyra \& Chaves, 2000; Lyra \& Rossetti-Ferreira, 1995; Lyra \& Souza, 2003; Lyra \& Winegar, 1997). Eles se distinguem pelas diferentes características das trocas diádicas. Essas trocas, sem a mediação do objeto (face-a-face, ou FF) ou com esta mediação (mãe-obje- 
to-bebê, ou MOB), exibem níveis desenvolvimentistas que possibilitam incluir aspectos generalizáveis e particulares presentes em cada díade mãe-bebê. São generalizáveis as formas assumidas pelas trocas que compõem um tipo característico de negociação entre os parceiros, o seu padrão de organização, e as particulares correspondem às ações específicas que cada díade escolhe para realizar essas trocas (Molenaar \& Valsiner, 2005). Ambos os aspectos resultam da história construída por cada díade. Este conjunto de três conceitos visa representar os processos subjacentes ao desenvolvimento do sistema de comunicação mãe-bebê, particularmente durante os primeiros oito meses de vida do bebê. As características do estabelecimento, da extensão e da abreviação estão descritas abaixo.

Estabelecimento: através de sucessivas ou concomitantes ações dos parceiros, que "recortam" ou destacam as atividades da díade, pelo menos um elemento das trocas diádicas é construído de forma partilhada pela díade. Este elemento se torna figura em relação a um fundo de outras ações realizadas pela díade. Por exemplo, o olhar mútuo entre os parceiros, considerando as trocas FF, ou o olhar dos parceiros para o objeto, considerando as trocas que envolvem objetos (MOB).

Extensão: o elemento ou os elementos previamente estabelecidos tornam-se um fundo em relação ao qual a díade pode "recortar" e negociar, elaborando de modo mais prolongado, outros elementos como figuras. Por exemplo, nas trocas FF, tendo estabelecido o contato de olhar mútuo, podem ser negociados sorrisos, vocalizações, movimentos, etc., de forma elaborada e prolongada. Considerando o olhar conjunto dos parceiros para o objeto como fundo, os movimentos dos braços/mãos do bebê para o objeto podem ser negociados durante trocas prolongadas que focalizam estes movimentos (nas trocas MOB).

Abreviação: trocas de curta duração executadas pela díade através de um ajustamento mútuo rápido e fácil, em que os elementos anteriormente negociados e trabalhados pela díade, de forma prolongada, aparecem de modo reduzido ou condensado (abreviados). Por exemplo, considerando as trocas FF, a díade utiliza um rápido contato de olhar acompanhado ou não de alguns elementos anteriormente negociados (sorriso, movimentos, por exemplo). Dando outro exemplo, tanto a mãe como o bebê, de forma ajustada, rápida e suave, dirigem o olhar para um objeto, que é então oferecido pela mãe e imediatamente segurado pelo bebê (nas trocas MOB).

Fundamentados na natureza construtiva do sistema de comunicação e, sobretudo, nas idéias do Dialogismo de Bakhtin $(1986,1993)$, propomos que na abreviação a díade exibe a emergência e diferenciação do bebê como sujeito ou self dialógico.

A seqüência de argumentos que se segue expõe, primeiramente, as posições ontológicas e epistemológicas que são as bases conceituais que fundamentam o modelo EEA. Destacamos, assim, os requisitos inerentes à investigação do processo histórico de desenvolvimento de um sistema de relações que possibilita a emergência do sujeito dialógico.
Discutimos também as possibilidades e os limites das duas posições teórico-metodológicas, Sistemas Dinâmicos e Dialogismo, que ancoram o referido modelo, destacando aspectos básicos da posição de Bakhtin $(1986 ; 1993)$ sobre o sujeito dialógico, ao mesmo tempo, relacional e único.

\section{Bases conceituais}

O termo modelo desperta diferentes expectativas. Segundo a concepção leiga, ele pode ser entendido como a "representação simplificada e abstrata de fenômeno ou situação concreta, e que serve de referência para a observação, estudo ou análise” (Ferreira, 1999). Num contexto científico, particularmente na Psicologia, podemos utilizar a palavra modelo tanto requerendo representações matemáticas (por exemplo, a álgebra booleana das estruturas cognitivas, segundo Piaget, ou os algoritmos que caracterizam a inteligência artificial, aplicados ao sistema binário computacional) como representando analogias de cunho qualitativo (por exemplo, a estrutura da personalidade, segundo Freud). Seja qual for a natureza do modelo, o seu papel consiste em fazer compreender, de forma simplificada, fenômenos que apresentam características complexas quando investigados nas suas variabilidades particulares e únicas de ocorrência (Overton, 1998).

Um modelo em qualquer ciência carrega, de forma explícita ou implícita, posições ontológicas e epistemológicas, presentes no que Pepper (1942) conceituou como hipóteses, metáforas ou posições sobre o mundo (world hypothesis). Essas posições, propostas por Pepper em 1942, ainda norteiam as diferentes correntes dominantes atuais na Psicologia. São quatro as posições por ele propostas: formismo, mecanicismo, organicismo e contextualismo.

O formismo se apóia na percepção que temos de um fenômeno composto de aspectos distintos que se apresentam freqüentemente unidos. Este fato faz sugerir a existência de formas típicas. Em Psicologia, um exemplo clássico está na caracterização da personalidade enquanto tipologias ou traços da personalidade - "introvertido versus extrovertido", por exemplo. Também, na tendência a diagnósticos que categorizam distúrbios ou desvios do comportamento ou do desenvolvimento humano, fornecendo a base para a abordagem das diferenças individuais enquanto classes estanques (Hermans \& Kempen, 1993). O mecanicismo encontra sua expressão nas perspectivas em Psicologia que compreendem a explicação do funcionamento humano através das relações entre antecedentes e conseqüentes. Essas relações estão expressas na concepção de causalidade presente no que Aristóteles chamou de causa eficiente. O exemplo clássico é o da bola de bilhar na qual a velocidade e a direção são causadas por outra bola que é concebida como causa eficiente. Em Psicologia, são tantos os exemplos do mecanicismo que não se faz necessário discorrer sobre este aspecto (Valsiner, 2001). O organicismo focaliza a maturação ou crescimento do organismo, destacando a direção, objetivo ou teleologia do comportamento ou do seu desenvolvimento. Utiliza-se da metáfora da causa final, ou thelos, usando uma linguagem Aristotélica, podendo incluir também a idéia de causa eficien- 
te, adotando ou não perspectivas construtivistas. Considerando as perspectivas desenvolvimentistas em Psicologia, pode-se ter como exemplo a teoria dos estágios de Piaget (que não é a teoria da equilibração). Finalmente, o contextualismo põe em destaque a natureza histórica dos eventos. O fenômeno humano é analisado e compreendido a partir de sua inserção no tempo e no espaço que compõem o contexto de sua realização (Hermans \& Kempen, 1993). Tratase de uma posição que destaca o caráter relacional e dinâmico do funcionamento humano, sobretudo do seu desenvolvimento. Os conceitos de historicidade e de contexto estão intimamente ligados, pois a natureza espacial do contexto fornece o caráter temporal da historicidade. A chamada segunda revolução cognitiva (Bruner, 1990) é o exemplo mais amplo e atual desta posição na Psicologia. A corrente interpretativista (Polkinghorne, 1988) é um outro exemplo atual na Psicologia.

A filiação de um modelo a uma única dessas "posições sobre o mundo" raramente é perfeita. Todavia, sempre existe uma ênfase na direção de uma dessas posições que se aplica às diferentes correntes da Psicologia nos nossos dias, com implicações sobre uma ontologia e uma epistemologia acerca do ser humano. Todavia, raramente o próprio cientista explicita a posição assumida acerca do fenômeno em estudo. Na sua maioria, o nosso sistema de formação de pesquisadores (as pós-graduações instituídas) toma por estabelecido que a pesquisa científica tenha um único, universal e eterno, por assim dizer, conjunto de pressupostos conceituais. Esses pressupostos fornecem as bases para a aprendizagem e aplicação do "método científico". Desta forma, o discernimento, pelo cientista, da sua "posição sobre o mundo" é estranho à formação científica estabelecida e consagrada presentemente. Todavia, esta posição guia a metodologia de coleta, análise e interpretação dos dados empíricos. Por exemplo, conceber o sujeito psicológico como resultado das relações entre antecedente e conseqüente retira deste sujeito a opção de ser autor da sua própria história. Este aspecto é possível, de alguma maneira, ao assumir uma posição advinda do contextualismo (mais especificamente, como nos propõe Bakhtin, 1986, 1993). Por outro lado, um extremo contextualismo pode dar margem à impossibilidade de generalizar que reconhecemos como necessária a todo empreendimento científico. A não explicitação das nossas hipóteses sobre o funcionamento do sujeito psicológico pode conduzir a incoerências marcantes ao adotar uma metodologia para coleta, análise e compreensão dos resultados de uma investigação científica.

O modelo EEA filia-se a uma "posição sobre o mundo" que destaca o caráter histórico, relacional, dinâmico e criativo do processo de desenvolvimento humano. Mais ainda, é o processo de comunicação que vai fazer emergir um sujeito psicológico único, diferenciado mas também relacional, capaz de assumir seu papel como autor da sua identidade. Explicitamos, assim, os fundamentos ontológicos e epistemológicos sobre os quais está baseado o modelo EEA.

\section{Processo histórico}

Estudar o processo de desenvolvimento requer que se investigue como as aquisições, ou produtos do desenvolvimento são integrados à dinâmica histórica que faz emergir tais aquisições. No entender de Valsiner (2001), todo e qualquer estudo do desenvolvimento requer este tipo de compromisso. Na literatura da Psicologia do Desenvolvimento este enfoque focaliza as mudanças que caracterizam o desenvolvimento (por exemplo, Fogel, 1993; Lee \& Kamiloff-Smith, 2002; Thelen \& Corbetta, 2002; Valsiner, 2001). Torna-se, então, imprescindível a inclusão do tempo como elemento integrante deste fenômeno. Isto quer dizer que cada elemento nele envolvido tem que ser considerado como sendo modificado - ou podendo ser modificado - porque é passível de transformação durante o transcorrer desse processo. Por exemplo, considerando o desenvolvimento da memória, das emoções ou da inteligência, o elemento conceituado como contexto social, mesmo definido clara e "operacionalmente", pode não ter o mesmo papel em diferentes faixas etárias (Bornstein \& Tamis-LeMonda, 2001) e mesmo em diferentes momentos da apresentação de um teste experimental (Vygotsky, 1987).

Este tempo, constitutivo do processo de desenvolvimento, é considerado como um tempo histórico (Lyra, 2000). A conceituação do caráter histórico do processo de desenvolvimento implica que as transformações que ocorrem carregam a abertura de possibilidades e o fechamento de outras possibilidades (Lyra \& Winegar, 1997). Esta abordagem processual e histórica reconhece e destaca a existência de um tempo irreversível que "deixa suas marcas" no desenrolar do processo de desenvolvimento, criando a história deste desenvolvimento e confundindo-se muitas vezes com o conceito de gênese. Todavia, a nossa compreensão é que a historicidade integra à concepção de gênese a projeção para o futuro necessária a todo sistema psicológico. Esta projeção é ao mesmo tempo baseada no passado, mas inclui a dimensão do futuro desconhecido. Trata-se de uma perene constituição do nosso presente com elementos do passado e também de um futuro imaginado. Vivemos no infinitamente mínimo momento do presente. Este momento é composto da seleção de porções do passado que têm a função de fornecer alguma previsibilidade para agir em um futuro desconhecido. Esta concepção tem raízes nas idéias de Bergson (1911) e tem sido elaborada por Valsiner (2002). Pelo menos em ciências humanas, temos que lidar com este aspecto inescapável, que se impõe, caracteristicamente, quando olhamos para a natureza semiótica do funcionamento humano (Valsiner, 2001, 2002; Vygotsky, 1986, 1987).

A ênfase no estudo das características do processo histórico de desenvolvimento, com a inclusão mais ou menos explícita desta projeção para o futuro, é um dos aspectos marcantes das abordagens que propõem que todo estudo da matéria viva se constitui em mudanças que ocorrem ao longo de um tempo irreversível (Baldwin, 1906; Edelman, 1987; Piaget, 1936/1953; Valsiner, 2002; Vygotsky, 1986). 


\section{Sistema de relações e indivíduo}

Um outro conceito que serve de base ao modelo EEA é o sistema de relações. A idéia de sistemas, definidos pelas relações entre seus elementos, não é nova em Psicologia (ver, por exemplo, Sameroff \& Chandler, 1975; Valsiner, 2001). Todavia, esta idéia nem sempre carrega, necessariamente, como unidade de análise a própria relação. Além do mais, qualquer perspectiva construtivista precisa enfrentar o dilema relação versus indivíduo ao conceituar a sua unidade de análise. Existe, de fato, uma forte tradição em Psicologia que situa o fenômeno do desenvolvimento como circunscrito, por assim dizer, “dentro” do indivíduo. Mesmo utilizando um discurso “interacionista”, portanto relacional, a metodologia ou método de pesquisa continua a ter um caráter ontológico individualista. Conceber as relações como unidade de análise é tarefa difícil. Marková (1987) relaciona esta dificuldade aos nossos hábitos ocidentais de pensamento que nos fazem encapsular a realidade em categorias distintas e estanques que nos distanciam da real dinâmica das relações que a realidade nos oferece (Gould, 1995).

Mais recentemente, a idéia de sistemas, particularmente dos Sistemas Dinâmicos em constante transformação, sobretudo no sentido da ênfase atribuída à natureza aberta desses sistemas (portanto, sujeitos a influências externas também), colocou em destaque a concepção das relações como unidade de análise. Considerando o sistema de comunicação, o trabalho de Fogel (1993) merece destaque especial.

No entanto, a tarefa de conceituar os sistemas em desenvolvimento não está completa. A idéia de sujeito, indivíduo, eu ou self continua a ser um requisito fundamental para a compreensão do ser humano. Ela está na base dos nossos papéis éticos e morais na sociedade que cria significado para o nosso funcionamento psicológico. Conseqüentemente, precisamos considerar, ao mesmo tempo, tanto as relações como os indivíduos, ambos emergindo de um mesmo processo de desenvolvimento. A necessidade de conceber tanto relações como indivíduos não é estranha nem mesmo aos sistemas físicos. Trata-se de um requisito de natureza ontológica que está na base desta aparente dualidade: a idéia de relação requer a existência de indivíduos em trocas dinâmicas, mesmo se considerarmos a realidade física do movimento browniano de partículas individuais interagindo em um fluído (ImpérioHamburger, Carvalho, \& Pedrosa, 2003).

Todavia, a noção de indivíduos e relações necessita considerar a natureza do sistema que possibilita a emergência e constituição de ambos de forma interdependente. Propomos que o sistema de comunicação seja o responsável pela construção de uma organização característica que possibilita a emergência de uma realidade virtual (Sinhá, 2004). O termo virtual aqui se aplica a um mundo, também real, mas que existe paralelo à nossa capacidade sensorial; uma duplicação transformada desta. É justamente na construção desta realidade dupla, por assim dizer, que emerge o indivíduo ou self. Propomos que esta construção tenha lugar nas relações comunicativas que se estabelecem desde o início da vida.
Assim, o sistema de comunicação focalizado pelo modelo EEA é um sistema de relações de natureza histórica, relacional, dinâmica e criativa que possibilita criar indivíduos ou selves. No seu estudo, fazemos uso de duas perspectivas teórico-metológicas: a perspectiva dos Sistemas Dinâmicos e a perspectiva que tem sido denominada Dialogismo (Holquist, 1990).

\section{Sistemas Dinâmicos e Dialogismo}

Como o sistema de comunicação faz emergir o ser humano? Mais especificamente, como faz emergir um sujeito psicológico, dotado da capacidade de "duplicar e reconstruir" o mundo através da sua capacidade semiótica? Faz-se necessário abordar os primórdios das características processuais que fazem emergir este sujeito. Este desafio também não é novo e não é específico às perspectivas referidas. Apenas para citar alguns dos grandes teóricos da Psicologia do Desenvolvimento, podemos relembrar que Piaget atribui esta capacidade à interiorização das coordenações sensóriomotoras, cujo advento primeiramente observável ocorre quando a criança começa a demonstrar uma imitação diferida, isto é, começa a reproduzir não imediatamente aspectos de uma situação ocorrida anteriormente (Piaget, 1936/1953). Vygotsky atribui à aquisição da linguagem a capacidade de interiorizar e emergir como semiótica a realidade do mundo que nos cerca. Assim, esta realidade é concebida como necessariamente social. Os períodos anteriores à linguagem são tratados por Vygotsky como característicos de uma "inteligência prática” que não teria características diferenciadoras das outras espécies que nos são próximas. A ausência da linguagem impossibilita, para Vygotsky, o funcionamento simbólico (Vygotsky, 1986, 1987).

\section{Por que Sistemas Dinâmicos? Processo de transformação, auto-organização e emergência do novo}

A compreensão do desenvolvimento como processo de transformação constitui um dos pontos centrais das idéias advindas da perspectiva dos Sistemas Dinâmicos, como nos destaca van Geert (1994). A partir dos anos 80 do século passado (ou, mais precisamente, a partir da segunda metade dos anos 1980), nenhuma perspectiva se dedicou tão explicitamente ao estudo do processo de transformação - ou da dinâmica que caracteriza o desenvolvimento - que aquela presentemente agrupada na Psicologia sob o rótulo de Sistemas Dinâmicos. A concepção do tempo como irreversível e a caracterização dos fenômenos como sistemas abertos, em constante troca de energia com o meio externo, mostraram-se revolucionárias no estudo de fenômenos físicos (Prigogine \& Stengers, 1984) e fenômenos biológicos (Edelman, 1987). Na Psicologia, as idéias decorrentes da perspectiva dos Sistemas Dinâmicos - também chamados de Sistemas Complexos ou Sistemas Caóticos (Thelen \& Smith, 1994) - progressivamente encontraram seu nicho (uma das primeiras propostas voltada para a Psicologia do Desenvolvimento acha-se em Fogel \& Thelen, 1987). Apesar de constituir uma abordagem 
recente, o seu campo de aplicação tem se expandido para diferentes áreas da Psicologia, dentre as quais destacamos, além da Psicologia do Desenvolvimento, a Psicopatologia e a Psicoterapia (Materpasqua \& Perna, 1997). Na Psicologia do Desenvolvimento, as concepções dos sistemas dinâmicos têm sido utilizadas para explorar o desenvolvimento motor (Thelen \& Smith 1994), o desenvolvimento cognitivo (Lewis, 1995; van Geert, 1994), o desenvolvimento do apego (Coleman \& Watson, 2000) e o desenvolvimento comunicativo e emocional (Fogel, 1993; Fogel \& Lyra, 1997; Lyra, 2000, no prelo, a, b e c; Lyra \& Rossetti-Ferreira, 1995; Lyra \& Souza, 2003; Lyra \& Winegar, 1997; Pantoja, 2001; Pantoja \& Nelson-Goens, 2000; Pantoja, Nelson-Goens, \& Fogel, 2001; Pedrosa, Carvalho, \& Império-Hamburger, 1997).

Um dos aspectos marcantes desta concepção está no conceito de auto-organização do sistema que possibilita a emergência de novas formas ou novos padrões de organização, cada vez mais complexos. Este mecanismo resulta das trocas entre os elementos que compõem o sistema de relações em estudo, possibilitando o desenvolvimento que é evidenciado através da emergência desses novos níveis de organização. A tarefa do investigador consiste no discernimento dos padrões de co-ação que definem as transformações e os níveis de organização de um dado sistema ao longo do tempo. Esta investigação permite a compreensão tanto dos períodos de maior estabilidade do sistema como dos períodos de instabilidade, que caracterizam as fases de mudança. Desta forma, na perspectiva dos Sistemas Dinâmicos, torna-se impossível (e sem sentido) a identificação de variáveis independentes e variáveis dependentes, uma vez que os elementos do sistema interagem e se modificam ao longo do tempo.

Está no conceito de atrator um dos aspectos mais importantes para compreender o processo de estabilidade e mudança dos Sistemas Dinâmicos. Um atrator - ou estado atrator - é concebido como uma região do sistema na qual existe uma tendência desse sistema para se estabilizar nesta região. O sistema apresenta, então, um comportamento de quase-estabilidade (Fogel \& Lyra, 1997; Lyra, 2000, Lyra \& Souza, 2003; Thelen \& Smith, 1994). Surgem todavia, novos atratores ao longo do tempo. Quando isto ocorre, o sistema sofre um processo de mudança, permanecendo instável durante certo período, até que, novamente, se estabiliza em um novo padrão de organização. Nestes períodos de instabilidade, diferentes atratores atuam ao mesmo tempo. Cada atrator representa uma opção de caminho - ou trajetória - que vai caracterizar o desenvolvimento futuro deste sistema. Considerando o modelo EEA, cada um dos padrões de organização propostos, que correspondem a períodos de quase-estabilidade - estabelecimento, extensão e abreviação - podem ser considerados como atratores que atuam no sistema de comunicação aqui estudado: as trocas mãe-bebê.

O mecanismo de passagem de um nível de organização para um outro padrão de organização guarda características de indeterminação (Fogel, Lyra, \& Valsiner, 1997). É justamente nessas passagens entre níveis de organização que emer- gem as novas formas ou novos padrões de organização. Existe, todavia, um jogo constante entre a determinação, proveniente da história de construção de um dado sistema, e a indeterminação, inerente ao momento de criação do novo (Lyra \& Winegar, 1997).

Embora os sistemas físicos e mesmo os sistemas biológicos possibilitem o uso de modelos matemáticos (Haken, 1983), na Psicologia a utilização dos conceitos provenientes da perspectiva dos Sistemas Dinâmicos tem sido realizada, sobretudo, de forma analógica (Thelen \& Smith, 1994). Uma exceção é o trabalho de van Geert $(1994,2002)$ que tem aplicado funções matemáticas para a simulação computacional do desenvolvimento da aprendizagem na relação professor-aluno, assim como para aspectos do desenvolvimento cognitivo e da linguagem. Outra exceção bastante inovadora encontra-se nas modelagens matemáticas propostas por Molenaar (Nesselroade \& Molenaar, 2003) para o processo de desenvolvimento.

\section{Por que Dialogismo? Sistema sócio-cultural de comunicação, emergência do novo e do self}

A idéia de diálogo está filiada a uma corrente de pensamento em ciências humanas que tem como requisito a existência de um sujeito psicológico (Marková, 1987, 1990). Isto quer dizer que o desenvolvimento ocorre, ao mesmo tempo, em um sistema de relações que adquirem níveis de organização cada vez mais complexos (resultantes de um processo de auto-organização). No entanto, este processo tem necessariamente que incluir a noção de sujeito psicológico ou self. Esta característica requer que pensemos conjuntamente em um fenômeno relacional e sistêmico, mas também construtor de indivíduos, sujeitos ou selves. O processo de diferenciação do sujeito humano exige um mecanismo de criação de uma realidade que se duplica e se reconstrói (uma realidade virtual, Sinhá, 2004). Esta realidade interiorizada é o que chamamos de capacidade simbólica ou semiótica. $\mathrm{O}$ sujeito psicológico humano é, ao mesmo tempo, um ser relacional e individual. Desta forma, o conceito de indivíduo, sujeito ou self é um requisito fundamental para uma compreensão do funcionamento psicológico humano e de seu desenvolvimento.

As concepções advindas da perspectiva dos Sistemas Dinâmicos não nos permitem abarcar esta dualidade necessária expressa na existência de relações e indivíduos. Utilizamos, da perspectiva dos Sistemas Dinâmicos, a investigação do processo de auto-organização do sistema em estudo. Por outro lado, fazemos uso das idéias elaboradas pelo Dialogismo, particularmente, o Dialogismo desenvolvido por Bahktin (1986, 1993), porque elas nos oferecem um instrumental que possibilita tratar o processo de emergência do sujeito psicológico ocorrendo no sistema de relações sociais desde o início da vida.

O trabalho do cientista que se propõe a investigar a emergência do sujeito humano necessita, primeiramente, caracterizar qual a natureza deste sistema de relações que possibilita a emergência desse sujeito. Trata-se de um requisito advindo 
da própria perspectiva adotada pelos teóricos dos Sistemas Dinâmicos: conhecer e delimitar a natureza do sistema em estudo (Thelen \& Smith, 1994; van Geert, 1994). A nossa resposta para esta caracterização é de que este sistema diz respeito ao processo de desenvolvimento da comunicação social humana desde os primeiros momentos de vida do bebê (Lyra, 2000; Lyra \& Rossetti-Ferreira, 1995; Lyra \& Winegar, 1997). Assim, o modelo EEA se aplica ao estudo do desenvolvimento da comunicação humana fazendo uso tanto de conceitos da perspectiva dos Sistemas Dinâmicos como daqueles que decorrem do Dialogismo proposto por Bakhtin.

Três aspectos, de natureza conceitual, caracterizam o sistema das relações sócio-culturais ou sócio-genéticas que compõe a comunicação - ou a troca dialógica - desde o início da vida: a participação da história cultural através da história ontogenética nas trocas dialógicas, o caráter relacional, histórico, interdependente e criativo do diálogo, e a diferenciação do indivíduo, eu ou self a partir deste diálogo.

Um dos pressupostos básicos das teorias sócio-genéticas, incluindo a maioria das interpretações ou conceituações atribuídas à perspectiva dialógica, é a sua dependência da linguagem (Marková, 1990), ou de um sistema simbólico, concebido como mediando as trocas do sujeito com o mundo social e físico que o cerca (Vygotsky, 1986). Este sistema de mediação simbólica, que resultou da história cultura1 da humanidade, constitui o sujeito psicológico humano, tal como o fez a filogênese em relação à espécie humana (Vygotsky \& Luria, 1994). Assim, quando falamos em comunicação dialógica tendemos a equipara-la à linguagem ou ao uso de um sistema simbólico já constituído.

Considerando, todavia, o início da vida, a questão que nos colocamos é, justamente, como o sistema de comunicação faz emergir e se desenvolver o sujeito psicológico humano que dispõe da linguagem e dos símbolos. Dois pressupostos nos guiam ao procurar investigar essa questão. Em primeiro lugar, concebemos que o bebê seja capaz de estabelecer uma relação dialógica anterior a qualquer linguagem (Bråten, 1988; Fogel, 1993; Fogel \& Lyra, 1997; Lyra, 2000; Lyra \& Winegar, 1997; Trevarthen, 1998). Propomos também que a história cultural da humanidade, refletida nos diversos níveis do ambiente físico e cultural-simbólico que circunda o bebê (espaço físico, normas, valores, atitudes, etc.), só se concretiza na relação comunicativa ou diálogo ao longo do tempo.

Começando com o segundo desses pressupostos, entendemos que a história cultural é necessariamente integrada e reconstruída ao criar o sujeito psicológico humano, através do sistema de comunicação ou diálogo, desde o início da vida. O sistema de comunicação aqui focalizado, aquele que compreende as trocas mãe-bebê, vai guardar limites e possibilidades culturalmente construídos que são inerentes a diferentes instâncias da história cultural. Alguns são mais gerais - por exemplo, aqueles característicos da linguagem - e alguns mais específicos - por exemplo, valores da nossa cultura ocidental; outros, ainda mais específicos, dizem respeito à história do grupo social, da família e da mãe/adulto que interage com o bebê (Lyra, 1998). Todavia, enfatizamos que, quaisquer que sejam estes limites e possibilidades, eles só se integram ao sistema de comunicação enquanto concretamente co-atuam nas trocas efetuadas entre os parceiros - no nosso caso o bebê e sua mãe - ao longo do tempo. Propomos, como caminho frutífero na análise sócio-genética, a investigação cuidadosa das características do processo de autoorganização do sistema de comunicação (períodos de quaseestabilidade e mudança) visando discernir os mecanismos processuais que fazem emergir as primeiras manifestações de uma mediação anterior ao símbolo lingüístico.

Desde o nascimento, o bebê acha-se inserido em uma dinâmica relacional, histórica, interdependente e criativa. Isto é o diálogo. Cada troca comunicativa é entendida como pertencente a ambos os parceiros, impossibilitando a separação de um emissor, de um receptor e da própria mensagem. Adotamos dessa forma uma perspectiva processual, dinâmica e contínua do processo de comunicação, que se opõe a uma visão discreta da comunicação (Fogel, 1993). Marková (1990) propõe que a unidade conceitual mínima de todo diálogo se circunscreva a três turnos: (1) a comunicação inicial de um dos parceiros, (2) a resposta do outro parceiro e (3) a resposta à resposta deste segundo parceiro, efetuada pelo primeiro. Este conceito de três turnos como unidade mínima do diálogo, condizente com o caráter criativo e transformador do diálogo, destaca a participação de cada parceiro no turno do outro, e a emergência de um produto sui generis como resultado do diálogo. Todavia, este conceito de três turnos deve ser guiado pela particular construção comunicativa, ou dialógica, em estudo. Neste sentido, os períodos de quaseestabilidade e mudança do sistema comunicativo, entendidos como produtos dialógicos, devem ser investigados à luz de uma unidade de análise que faça compreender a história de construção destes períodos. Esta unidade pode incluir turnos passados não contidos nos três referidos turnos sugeridos por Marková. Desta forma, é a natureza da aquisição comunicativa que guia o quanto se faz necessário retornar na história passada particular de cada díade. Para compreender a natureza criativa do diálogo, os turnos entre os parceiros são considerados como contidos em um todo histórico maior, cuja dimensão é guiada pela natureza da construção específica que é focalizada (Lyra, 1999).

Tanto a perspectiva dos Sistemas Dinâmicos, porque aceita a mútua transformação dos elementos que compõem o sistema em desenvolvimento, como a perspectiva Dialógica, concebem a coexistência de contradições como inerentes aos sistemas em desenvolvimento. Marková (1987) faz uma excelente análise da dificuldade enfrentada pelos estudiosos do desenvolvimento da interação por se apegarem ao princípio da não-contradição. Este princípio, remontando ao pensamento de Aristóteles e posteriormente de Kant, reza que um dado fenômeno não pode ser, ao mesmo tempo, A e não-A. Por exemplo, analisando o desenvolvimento como resultante da interação do organismo com o meio, o princípio da nãocontradição tanto não permite que se considere como coexistindo fatores inerentes ao organismo e ao ambiente como 
impossibilita a consideração do processo de mudança. A nãocontradição admite apenas a passagem de um ponto estático para outro, no qual o movimento se reduz a uma sucessão de estados estáticos. O processo é assim excluído como possibilidade ontológica.

Fundamentado neste princípio ontológico, os estudiosos do desenvolvimento da comunicação excluem a possibilidade de que a relação comunicativa seja a condição na qual as aquisições do desenvolvimento pertencem, ao mesmo tempo, a cada sujeito e à relação dialógica que, necessariamente, inclui o parceiro. Este caráter de interdependência de cada parceiro social na relação dialógica é o primeiro aspecto que define a natureza específica dos sistemas de relações sociais que caracterizam o diálogo e seu desenvolvimento. Vamos agora descrever a coexistência da relação e dos parceiros a partir das idéias de Bakhtin.

\section{O sujeito dialógico segundo Bakhtin}

Dois aspectos do Dialogismo proposto por Bakhtin (1986, 1993) são extremamente relevantes para o modelo EEA, no que se refere à emergência e constituição do self: a existência de um único lugar - no tempo e no espaço - possível de ser ocupado pelo sujeito e a característica de responsividade (answerability).

Nessa perspectiva, a idéia de indivíduo está baseada em um axioma que estabelece a existência de um único lugar no mundo onde existe um sujeito. Este lugar - espacial e temporal - só é ocupado por uma única pessoa no transcorrer da autobiografia da sua vida. Esta autobiografia é escrita por cada indivíduo como autor. O sujeito é autor de sua própria história, simplesmente porque não há outra possibilidade para que ele exista. Sem autoria não há diálogo possível no sentido genuíno da troca criativa que caracteriza o diálogo. Apenas desta perspectiva o diálogo é constitutivo da pessoa humana. Assim é que se distingue e se aplica o Dialogismo.

Bakhtin também propõe uma idéia complementar. Do lugar único que cada um ocupa, só existe uma possibilidade de existência, a inescapável necessidade de responder ao mundo que nos cerca (responsividade). Bakhtin utiliza a expressão "não temos álibi possível”, porque responder ao mundo que nos cerca não é uma escolha, mas uma condição para existir (Bakhtin, 1989, citado por Holquist, 1990, p. 29). Ainda, este mundo ao qual respondemos é um mundo de outros que nos respondem também porque o diálogo é absolutamente pervasivo. Tornando mais claro, este mundo é o mundo social - um mundo de selves - característico da espécie humana.

Considerando o modelo EEA, estes dois aspectos do Dialogismo servem de base para a análise das características do padrão de organização, denominado abreviação. A abreviação identifica uma organização do diálogo mãe-bebê na qual se vislumbra um parceiro bebê começando a exibir um grau primeiro de diferenciação como sujeito. A qualidade das trocas abreviadas possibilita inferir a existência de uma nova realidade, uma realidade virtual na qual o bebê começa a distinguir a intenção comunicativa do parceiro daquela resultante, apenas, da aprendizagem contingente das ações. Nes- ta realidade emergente, o bebê ocupa um único lugar possível, evidente a partir da análise da história de construção do diálogo. Deste lugar, ele responde ao outro, mesmo que nada faça, e este outro, de forma inescapável, lhe responde também.

\section{Conclusão}

A necessidade de uma integração explícita entre aspectos teóricos e metodologia é uma exigência para a avaliação da natureza, do valor e do papel da nossa contribuição à ciência e à sociedade mais ampla em que vivemos. Partindo dos seus objetivos e da proposta inicial, procuramos apresentar, discutir e justificar as bases conceituais e teóricometodológicas que fundamentam o modelo EEA. Estamos propondo uma integração das contribuições provenientes da perspectiva dos Sistemas Dinâmicos e do Dialogismo, que se apóia no caráter histórico, relacional, dinâmico e criativo do processo do desenvolvimento humano. Propomos também que o processo de comunicação seja o locus de onde o indivíduo, sujeito ou self é criado como parceiro do diálogo que o constitui.

As características empíricas do modelo EEA, incluindo as definições e ilustrações dos padrões de organização denominados de estabelecimento, extensão e abreviação, a apresentação da unidade de análise e algumas aplicações sugeridas, compõem um outro trabalho que apresenta a aplicação deste modelo para o estudo do desenvolvimento do processo de comunicação mãe-bebê e da emergência do self (Lyra, no prelo b).

\section{Agradecimentos}

Agradecimentos ao $\mathrm{CNPq}$ - projeto integrado de pesquisa/bolsa de produtividade, processo 523550/96-0, e aos colegas, colaboradores e amigos, Maria Clara F. Batista, Sílvio R. A. Salinas, Vilma A. M. de Queiroz, Letícia S. Galembeck e Emmanuelle C. Chaves, que fizeram sugestões valiosas a partir da leitura de versões preliminares deste artigo.

\section{Referências}

Bakhtin, M. (1986). Speech genres and other late essays (C. Emerson \& M. Holquist, Orgs.; V. W. McGee, Trad.). Austin: University of Texas Press. Bakhtin, M. (1993). Toward a philosophy of the act (M. Holquist \& V. Liapunov, Orgs.; V. Liapunov, Trad.) Austin: University of Texas Press.

Baldwin, J. M. (1906). Thought and things: a study of the development and meaning of thought, or genetic logic: Vol. I. Functional logic, or genetic theory of knowledge. Londres: Swan Sonnenschein.

Bergson, H. (1911). Creative evolution. Nova York: Henry Holt.

Bornstein, M. H., \& Tamis-LeMonda, C. S. (2001). Mother-infant interactions. In G. Bremner \& A. Fogel (Orgs.), Infant development (pp. 269-295). Oxford: Blachwell.

Bråten, S. (1988). Dialogic mind: the infant and the adult in protoconversation. In M. Carvalho (Org.), Nature, cognition and system (pp. 187-205). Dordrecht, Holanda: Springer.

Bruner, J. (1990). Acts of meaning. Cambridge, Massachusetts: Harvard University Press. 
Coleman, P., \& Watson, A. (2000). Infant attachment as a dynamic system. Human Development, 43, 295-313.

Edelman, G. M. (1987). Neural darwinism. Nova York: Basic Books.

Ferreira, A. B. H. (1999). Novo Aurélio século XXI: o dicionário da língua portuguesa/Aurélio Buarque de Holanda Ferreira ( $3^{\underline{a}}$ ed.). Rio de Janeiro: Nova Fronteira.

Fogel, A. (1993). Developing through relationships. Origins of communication, self and culture. Chicago: University of Chicago Press.

Fogel, A., \& Lyra, M. C. D. P. (1997). Dynamics of development in relationships. In F. Masterpasqua \& P. Perna (Orgs.), The psychological meaning of chaos: translating theory into practice (pp. 75-94). Washington, DC: American Psychological Association.

Fogel, A., Lyra, M. C. D. P., \& Valsiner, J. (1997). Introduction: perspectives on indeterminism and development. In A. Fogel, M. C. D. P. Lyra, \& J. Valsiner (Orgs.), Dynamics and indeterminism in developmental and social processes (pp. 1-10). Mahwah, New Jersey: Lawrence Erlbaum.

Fogel, A., \& Thelen, E. (1987). Development of early expressive and communicative action: reinterpreting the evidence from a dynamic system perspective. Developmental Psychology, 23, 747-761.

Gould, S. J. (1995). Dinosaur in a haystack. Nova York: Harmony Books.

Haken, H. (1983). Synergetics an introduction: non-equilibrium phase transitions and self-organization in physics, chemistry, and biology. Berlin: Springer.

Hermans, H. J. M., \& Kempen, H. J. G. (1993). The dialogical self. Meaning as movement. Nova York: Academic.

Holquist, M. (1990). Dialogism. Bakhtin and his world. Londres: Routledge.

Império-Hamburger, A., Carvalho, A. M. A., \& Pedrosa. M. I. (2003). Movimentos ao acaso e princípios de ordenação em grupos de crianças brincando. In T. Tomé (Org.), Tendências da física estatística no Brasil (pp. 105-109). São Paulo: Editora Livraria da Física.

Lee, K., \& Karmiloff-Smith, A. (2002). In N. Granott \& J. Parziale (Orgs.), Microdevelopment. Transition processes in development and learning (pp. 243-265). Cambridge: Cambridge University Press.

Lewis, M. D. (1995). Cognition-emotion feedback and self-organization of developmental paths. Human Development, 38, 71-102.

Lyra, M. C. D. P. (1998). Reflections on the dynamics of meaning making: communication process at the beginning of life. In M. C. D. P. Lyra \& J. Valsiner (Orgs.), Construction of psychological processes in interpersonal communication (pp. 225-242). Norwood, New Jersey: Ablex.

Lyra, M. C. D. P. (1999). An excursion into the dynamics of dialogue: a rejoinder to the Dialogical Self (by Hermans and Kempen). Culture \& Psychology, 5(4), 477-489

Lyra, M. C. D. P. (2000). Desenvolvimento como processo de mudança em um sistema de relações historicamente construído: contribuições do estudo da comunicação no início da vida. Psicologia: Reflexão e Crítica, 13(2), 257-268.

Lyra, M. C. D. P. (no prelo a). Desenvolvimento como processo de mudança. In A. G. Spinillo \& L. L. Meira (Orgs.), Psicologia cognitiva: cultura, desenvolvimento e aprendizagem. Recife: Editora da Universidade Federal de Pernambuco.

Lyra, M. C. D. P. (no prelo b). O modelo EEA: definições, unidade de análise e possíveis aplicações. Psicologia: Reflexão e Crítica.

Lyra, M. C. D. P. (no prelo c). Mother-infant communication development and the emergence of self: the contributions of dynamic systems and dialogism. In C. Lightfoot, M. C. D. P. Lyra, \& J. Valsiner (Orgs.), Challenges and strategies for studying human development in cultural contexts. Greenwich, Connecticut: Information Age.

Lyra, M. C. D. P., \& Chaves, E. C. (2000). O desenvolvimento da comunicação no início da vida: estabelecimento, extensão e abreviação. Temas em Psicologia, 8(3), 225-240.

Lyra, M. C. D. P., \& Rossetti-Ferreira, M. C. (1995). Transformation and construction in social interaction: a new perspective on analysis of the mother-infant dyad. In J. Valsiner (Org.), Child development within culturally structured environments (pp. 51-77). Norwood, New Jersey: Ablex.

Lyra, M. C. D. P., \& Souza, M. (2003). Dynamics of dialogue and emergence of self in early communication. In I. Josephs (Org.), Dialogicality in development (pp. 51-68). Londres: Praeger.

Lyra, M. C. D. P., \& Winegar, L. T. (1997). Processual dynamics of interactions through time: adult-child interactions and process of development. In A. Fogel, M. C. D. P. Lyra, \& J. Valsiner (Orgs.), Dynamics and indeterminism in developmental and social processes (pp. 93-109). Mahwah, New Jersey: Lawrence Erlbaum.

Marková, I. (1987). On the interaction of opposites in psychological processes. Journal for the Theory of Social Behaviour, 17, 279-299.

Marková, I. (1990). A three-step process as a unit of analysis in dialogue. In I. Marková \& K. Foppa (Orgs.), The dynamics of dialogue (pp. 129-146). Nova York: Springer-Verlag.

Masterpasqua, F., \& Perna, P. (Orgs.). (1997). The psychological meaning of chaos: translating theory into practice. Washington, DC: American Psychological Association.

Molenaar, P. C. M., \& Valsiner, L. (2005). How generalization works through the single case: a simple idiographic process analysis of an individual psychotherapy. International Journal of Idiographic Science, 1. Obtido de http://www.valsiner.com/ articles/molenvals.htm.

Nesselroade, J. R., \& Molenaar, P. C. M. (2003). Quantitative models for developmental processes. In J. Valsiner \& K. Connolly (Orgs.), Handbook of developmental psychology (pp. 622-639). Londres: Sage.

Overton, W. F. (1998). Developmental psychology: philosophy, concepts, and methodology. In R. M. Lerner (Org.), Theoretical models of human development. Vol. 1 Handbook of child psychology (pp. 107-188). Nova York: Wiley.

Pantoja, A. P. F. (2001, Setembro). A narrative-developmental approach to early emotions. Forum: Qualitative Social Research, 2(3). Disponível em http:/ /www.qualitative-research.net/fqs-texte/3-01/3-01pantoja-e.htm.

Pantoja, A. P. F., \& Nelson-Goens, G. C. (2000). Desenvolvimento da vida emocional durante o segundo ano de vida: narrativas e sistemas dinâmicos. Psicologia: Reflexão e Crítica, 13(2), 269-280.

Pantoja, A. P. F., Nelson-Goens, G. C., \& Fogel, A. (2001). A dynamical systems approach to the study of early emotional development in the context of mother-infant communication. In A. F. Kalverboer \& A. Gramsbergen (Ogs.), Brain and behavior in human development (pp. 901-920). Dordrecht, Holanda: Kluwer.

Pedrosa, M. I., Carvalho, A. M. A., \& Império-Hamburger, A. (1997). From disordered to ordered movement: attractor configuration and development. In A. Fogel, M. C. D. P. Lyra, \& J. Valsiner (Orgs.), Dynamics and indeterminism in developmental and social processes (pp. 135-151). Mahwah, New Jersey: Lawrence Erlbaum.

Pepper, S. C. (1942). World hypothesis. Berkeley, Califórnia: University of California Press.

Piaget, J. (1936/1953). Origins of intelligence in the child. Londres: Routledge \& Kegan

Polkinghorne, D. (1988). Narrative knowing and the human sciences. Nova York: State University of New York Press.

Prigogine, I., \& Stengers, I. (1984). Order out of chaos: man's new dialogue with nature. Nova York: Bantam Books.

Sameroff, A. J., \& Chandler, M. J. (1975). Reproductive risk and continuum of caretaking casuality. In F. D. Horowitz, M. Hetherington, S. Scarr-Salapetck, \& G. Siegal (Orgs.), Review of child development research. Vol. 4 (pp. 187224). Chicago: University of Chicago Press.

Sinhá, C. (2004, novembro). Practice, process and sign in meaning construction. Comunicação apresentada no Third Serrambi Meetings, Methods for Studying Human Development in Culturally Diverse Contexts, Serrambi, PE, Brasil. 
Thelen, E., \& Corbetta, D. (2002). Microdevelopment and dynamic systems: apllications to infant development. In N. Granott \& J. Parziale (Orgs.), Microdevelopment. Transition processes in development and learning (pp.5979). Cambridge: Cambridge University Press.

Thelen, E., \& Smith, L. B. (1994). A dynamic system approach to the development of cognition and action. Cambridge, Massachusetts: The MIT Press.

Trevarthen, C. (1998). The concept and foundations of infant intersubjectivity. In S. Bråten (Org.), Intersubjective communication and emotion in early ontogeny (pp. 15-46). Cambridge: Cambridge University Press.

Valsiner, J. (2001). Comparative study of human cultural development. Madri: Fundacion Infancia y Aprendizage.

Valsiner, J. (2002). Irreversibility of time and ontopotentiality of signs. Estudios de Psicologia, 23(1), 49-59.

Valsiner, J. (2006). Developmental epistemology and implications for methodology. In W. Damon \& R. M. Lerner (Orgs.), Handbook of child psychology. Theoretical models of human development (pp. 166-209). Haboken, New Jersey: John Wiley \& Sons.

van Geert, P. (1994). Dynamic systems of development: Change between complexity and chaos. Nova York: Harvester.

van Geert, P. (2002). Developmental dynamics, intention actions and fuzzy sets. In N. Granott \& J. Parziale (Orgs.), Microdevelopment. Transition processes in development and learning (pp. 319-343). Cambridge: Cambridge University Press.

Vygotsky, L. S. (1986). Thought and language. Cambridge, Massachusetts: The MIT Press.

Vygotsky, L. S. (1987). The collected work of L. S. Vygotsky. Vol. 1. Problems of general psychology. Nova York: Plenum.

Vygotsky, L., \& Luria, A. (1994). Tool and symbol in child development. In R. van der Veer \& J. Valsiner (Orgs), The Vygotsky reader (pp. 99-174). Cambridge, Massachusetts: Blackwell.

Maria da Conceição Diniz Pereira de Lyra, doutora em Psicologia Experimental pela Universidade de São Paulo, é professora e pesquisadora na Universidade Federal de Pernambuco. Endereço para correspondência: Rua Muniz Tavares, 147/1001, Jaqueira, 52050-170, Recife, PE. E-mail: marialyra2005@yahoo.com.br 